Pedagogía y Saberes No. 49

Universidad Pedagógica Nacional

Facultad de Educación. 2018, pp. 27-40

\section{La práctica pedagógica como herramienta para historiar la pedagogía en Colombia*}

Artículo de investigación

Pedagogy in Colombia A prática pedagógica: ferramenta para historicizar a pedagogia na Colômbia

Rafael Ríos Beltrán"

Para citar este artículo:

Ríos, R. (2018). La práctica pedagógica como herramienta para historiar la pedagogía en Colombia. Pedagogía y Saberes, 49, 27-40.

* Las siguientes reflexiones son resultado del proyecto de investigación "Miradas sobre la configuración de los saberes y disciplinas escolares en Colombia” financiado por el coDI de la Universidad de Antioquia.

** Profesor del Instituto de Educación y Pedagogía de la Universidad del Valle. Doctor en Educación y pedagogía e investigador del Grupo de Historia de la Práctica Pedagógica-GHPP.

Correo electrónico: rafael.rios@correounivalle.edu.co

Código ORCID: http://orcid.org/0000-0001-8049-1986 


\title{
Resumen
}

La indagación histórica acerca de la práctica pedagógica en Colombia es un trabajo metodológico y conceptual que ha posibilitado el develamiento del maestro como sujeto de saber pedagógico y la postulación de la enseñanza como un objeto y una práctica de saber, con lo cual se supera su mirada instrumentalista. Este artículo de investigación pretende mostrar el contexto a través del cual surge la noción práctica pedagógica, al interior del Grupo Historia de la Práctica Pedagógica (GHPP), no como una experiencia académica acabada, sino como una experiencia de investigación en el campo de la pedagogía en Colombia

\section{Palabras clave}

práctica pedagógica; pedagogía en Colombia; historia de la pedagogía; enseñanza

\begin{abstract}
Historical research on pedagogical practice in Colombia is a methodological and conceptual work that has enabled the unveiling of the teacher as a subject of pedagogical knowledge and the postulation of teaching as an object and practice of knowledge, which has made it possible to move beyond an instrumentalist view. The research paper aims to show the context through which the pedagogical practical notion emerges in the History of Pedagogical Practice Group (GHPP), not as a finished academic experience but as a research experience in the field of pedagogy in Colombia.
\end{abstract}

\section{Keywords}

pedagogical practice; pedagogy in Colombia; history of pedagogy; teaching

\section{Resumo}

A pesquisa histórica em torno da prática pedagógica na Colômbia é um trabalho metodológico e conceitual que possibilita a compreensão do professor como sujeito do saber pedagógico e a postulação do ensino como objeto e como prática do saber, perspectiva que supera o aspecto instrumental. 0 artigo mostra o contexto através do qual a noção prática pedagógica surge, não como uma experiência acadêmica terminada, mas como experiência de pesquisa dentro do grupo História da prática pedagógica (GHPP), no campo da pedagogia na Colômbia.

\section{Palavras-chave}

prática pedagógica; história da pedagogia; pedagogia na Colômbia; ensino 


\section{Introducción}

El Grupo Historia de la Práctica Pedagógica (GHPP) inicia sus actividades investigativas en 1978, fecha que marca el comienzo de los trabajos pioneros que conforman el actual acerbo conceptual, teórico y metodológico de este grupo de investigación en Colombia.

El GHPP se conforma en torno a preocupaciones comunes acerca de la pedagogía, su historia, la historia de la educación, el análisis de los pedagogos y la inscripción de sus aportes para tematizar las problemáticas que afectan al maestro, la enseñanza y la escuela, es decir, a los tres elementos constitutivos del maestro, reunidos bajo la expresión práctica pedagógica como una noción metodológica que conjuga tres categorías: el saber, el sujeto y la institución, que a su vez se inscriben en la noción de saber pedagógico.

El escrito se desarrolla en cuatro apartados: el primero describe brevemente el acercamiento que realizó la profesora Olga Lucia Zuluaga a la obra de Michel Foucault para precisar un objeto de investigación desde el campo de la pedagogía, lo cual dio lugar a la noción práctica pedagógica y saber pedagógico. El segundo apartado ofrece una lectura del libro Pedagogía e historia (1987) de Zuluaga para hacer visible el propósito fundamental del GHPP, a saber, rescatar la práctica pedagógica para recuperar la historicidad de la pedagogía. El tercer apartado se ocupa de las nuevas lecturas que sobre este concepto han realizado algunos investigadores y miembros del GHPP. Finalizamos con algunas reflexiones sobre el uso de esta herramienta metodológica para pensar nuevos programas de investigaciones sobre la pedagogía en Colombia.

\section{Los inicios}

En 1975, la profesora Olga Lucia Zuluaga comienza una experiencia de investigación de la obra de Michel Foucault en dos direcciones: la primera se dirigió a los problemas fundamentales de su trabajo y la segunda estuvo encaminada a precisar un objeto de investigación en el campo de la pedagogía. De esta manera, se inicia una lectura filosófica y temática de sus libros publicados hasta ese momento por el pensador francés, entre 1962 y 1970, a saber: Historia de la locura, El nacimiento de la clínica, Las palabras y las cosas, La arqueología del saber y el Orden del discurso.

De este acercamiento a la obra de Foucault surge el proyecto "Filosofía y pedagogía" desarrollado entre 1975 y 1978. Este consta de tres volúmenes, publicados por el Centro de Investigaciones Educativas de la Facultad de Educación de la Universidad de
Antioquia: Filosofía y pedagogía (1976), Didáctica y conocimiento (1977) y Colombia: dos modelos de su práctica pedagógica en el siglo XIX (1978).

El encuentro de Zuluaga con la obra del pensador francés se confrontó simultáneamente con las fuentes primarias de la pedagogía, cuya presencia en la investigación y la docencia en nuestro país era bastante débil y evocaba, entre otras cosas, un pasado inútil. Desde ese encuentro, los trabajos de Foucault abrieron la posibilidad de análisis tanto arqueológicos como genealógicos con orientación epistemológica, los cuales son capaces de dar cuenta de la formación de objetos, conceptos y técnicas en la interioridad de prácticas sociales.

En consecuencia, la obra de Foucault permitió, en relación con los pensadores clásicos de la pedagogía (Vives, Comenio, Rousseau, Pestalozzi, Herbart, Claparède), construir dos nociones: saber pedagógico y práctica pedagógica, especificando así un trabajo de investigación de tipo histórico que analizara la cultura y las prácticas de saber en las instituciones y los sujetos en Colombia. De esta manera, cuando la profesora Zuluaga convoca al Grupo de Historia de la Práctica Pedagógica en 1978, el trabajo de investigación pretendía:

- Dar una mirada epistemológica desde los terrenos arqueológicos a los discursos sobre la educación y la pedagogía en las facultades de educación y escuelas normales de nuestro país.

- Vincular los estudios de historia de la educación a la historia social del país y encontrar un nuevo punto de vista diferente a la presencia de la ideología dominante y del aparato ideológico de corto alcance para analizar los fenómenos internos y externos de la escuela.

- Abrir un campo de confluencia entre una cultura, un pensamiento, una pedagogía y un modelo o método pedagógico vinculados a prácticas históricas que podían tener un significado para el presente. (Zuluaga, Echeverri y Quiceno, 1999, p. 5).

Con la "caja de herramientas Foucault" el grupo inicia la construcción de una metodología que permitió el desplazamiento de una historia de las ideas pedagógicas hacia una historia de las prácticas y las conceptualizaciones pedagógicas. Tal movimiento plantea que

No es posible concebir, desde un punto de vista arqueológico o genealógico, el discurso por una parte, y las prácticas por otra. No es posible situarse por fuera del discurso para analizar las prácticas. A través de los discursos se conocen las prácticas, es decir, se hacen accesibles. (Zuluaga, 1987, p. 114). 
Esto aplicado a la pedagogía conduce a "No hacer la separación entre la práctica y la conceptualización, es una manera diferente de hacer historia que rompe por completo con las viejas preguntas de la historia de la pedagogía" (Zuluaga, 1997, p. 23).

En ese sentido, se construyó un vocabulario metodológico para analizar las prácticas de saber. Los documentos se acogieron como registro de una práctica y no como testimonio silencioso que debe llenarse de contenidos desde otra discursividad. La palabra $\operatorname{archivo}^{1}$ reemplazó aquella masa documental inerte que guarda la memoria de lo dicho. La teoría, la ciencia o la ideología se nombró como saber ${ }^{2}$, el lenguaje cotidiano o especializado como discurso ${ }^{3}$, y las determinaciones o las aplicaciones como práctica ${ }^{4}$.

Según lo anterior, resulta claro que la forma de trabajo de la obra de Foucault no fue ni la del comentarista, ni la del apologista que, en general, define a los foucaultianos ortodoxos, sino más bien como el de "caja de herramientas", tan preciado por él mismo. Desde esta noción, el grupo se dirigió a otras teorías y herramientas metodológicas distintas a la de Foucault, pero ante todo leyendo nuestra propia sociedad y cultura. Entre esas herramientas de varios campos están:

- La historia epistemológica del francés, Georges Canguilhem. En él se encontraron herramientas para reconocer, "[...] en la historicidad de los saberes, cuándo un discurso alcanza el estatuto de ciencia con sus elementos constitutivos a saber: el objeto con su correspondiente método y los conceptos" (Zuluaga, 1987, p. 17).

- La historia de la pedagogía y la educación. Este conjunto de datos, biografías, apartes sobre legislación educativa y de historias de las ideas fue una fuente obligada para el trabajo histórico-crítico de la pedagogía.

1 El archivo para este grupo de investigación fue, en la expresión de Foucault, la "ley de lo que puede ser dicho" (1996a, p. 219).

2 Conjunto de conocimientos de niveles desiguales (cotidianos o con pretensión de teóricos), cuyos objetos son enunciados en diversas prácticas y por sujetos diferentes.

3 Concepto central en Foucault para incluir en la historia los aspectos sociales. Se refiere a lo que puede ser dicho y pensado, son prácticas que configuran sistemáticamente los objetos de los que hablan.

4 Las prácticas poseen una realidad efectiva, se definen por el saber que forman y son susceptibles de ser historiadas. Además, las prácticas de las instituciones tienen por finalidad la circulación, la difusión, la producción, la adecuación, la distribución y el control de saberes.
- Las fuentes primarias de la pedagogía. Volver la mirada a los pedagogos clásicos permitió aprehender los enunciados más internos del saber pedagógico, definir el maestro como el sujeto del saber pedagógico y delimitar un campo de relación de la pedagogía con otros saberes.

También, bajo la noción caja de herramientas se estudiaron y analizaron a historiadores colombianos, como Jaime Jaramillo, Germán Colmenares y Marco Palacios, e historiadores extranjeros que han historiado a Colombia, como Aline Helg, Malcom Deas, David Bushnell y Frank Safford.

En suma, la obra de Foucault fue apoyo y herramienta conceptual importante para el trabajo histórico de la pedagogía como práctica y como saber en Colombia, de ahí que desde sus inicios el proyecto "Hacia una historia de la práctica pedagógica en Colombia"5 fuese planteado como historia de las prácticas ${ }^{6}$, las instituciones y los sujetos comprometidos en el funcionamiento del saber pedagógico. De esta manera, se realiza un trabajo histórico sin precedentes en Colombia, una nueva historia de la pedagogía: una historia desde la práctica pedagógica.

\section{Pedagogía e historia: una lectura del libro de Zuluaga}

En 1987, surge un trabajo que retoma los análisis sobre el maestro, la institución educativa y la formación del saber pedagógico en Colombia, nos referimos al libro Pedagogía e historia. La historicidad de la pedagogía. La enseñanza un objeto de saber. Esta obra comprende la segunda etapa del proyecto "Hacia una historia de las prácticas pedagógicas en Colombia". Es un alto en el camino para evaluar y refinar no solo la metodología puesta a prueba en este proyecto, sino también las conceptualizaciones sobre la pedagogía. En él, encontraremos nuevos desarrollos y comprensiones tanto sobre la noción que nos ocupa

5 Este programa de investigación interuniversitario estuvo compuesto por la Universidad Nacional de Colombia, la Universidad del Valle, la Universidad Pedagógica Nacional y la Universidad de Antioquia como coordinadora de este programa. Consta de cuatro proyectos: "Los jesuitas como maestros", "La práctica pedagógica durante la Colonia”, "La práctica pedagógica del siglo XIX”, "La práctica pedagógica del siglo Xx". Colciencias (entidad gubernamental que financia la investigación en Colombia) en 1980 apoyó financieramente este proyecto.

6 El trabajo histórico y crítico de la pedagogía en nuestro país no ha de entenderse como una simple historia de prácticas, de formas de enseñanza. La historia de las prácticas realizada en las investigaciones del GHPP tiene que ver con el modo de historiar los saberes desde la tríada metodológica: instituciones, sujetos y discursos. 
en este escrito: la práctica pedagógica, como sobre los conceptos que forman una "red conceptual" a su alrededor, y los argumentos para desarrollar interrogantes como: ¿qué es lo que permite historiar la práctica pedagógica?, ¿qué es lo que hace visible para análisis epistemológicos?, ¿por qué práctica pedagógica y no práctica educativa, práctica de enseñanza o práctica de formación?, ¿cómo está ahí el maestro y cómo el saber pedagógico?, entre otros interrogantes. Tratemos de abrir las páginas de esta obra.

Según lo planteado por Zuluaga, el propósito fundamental es rescatar la práctica pedagógica, en primer lugar, para recuperar la historicidad de la pedagogía. Este proyecto de recuperación exige analizar la pedagogía como saber y como disciplina en formación. En segundo lugar, se trabaja con el discurso de la pedagogía, ya que es su condición de posibilidad,

[...] pues al abordar el análisis de un discurso y de su práctica (práctica pedagógica), en su condición de saber (modalidad de análisis), la delimitación del sujeto de saber (el maestro) y del saber apropiado, no se puede plantear por fuera del saber (la pedagogía), porque ella involucra tanto al sujeto del saber como al saber mismo. (Zuluaga, 1987, p. 24).

En tercer lugar, se analiza la práctica del saber pedagógico en nuestra sociedad. La práctica pedagógica, en la perspectiva de historiar los saberes, asume el discurso de la pedagogía con "pretensión teórica y sistemática”, de ahí su carácter estratégico en la metodología, pues caracteriza el objeto de análisis (la pedagogía) como un objeto histórico complejo. Pero, "no se trata de una estructura, se trata de un proceso susceptible de abordar, desde diferentes modalidades, en la perspectiva de la historia de los saberes" (Zuluaga, 1987, p. 30).

¿Qué permite registrar históricamente la práctica pedagógica? Desde la herramienta Foucault, podemos afirmar que sería el umbral de positividad de la pedagogía. Por este hemos de entender el momento

a partir del cual una práctica discursiva (o práctica pedagógica) se individualiza y adquiere su autonomía, al momento, por consiguiente, en que se encuentra actuando un único sistema de formación de los enunciados, o también al momento en que ese sistema se transforma ${ }^{7}$. (Foucault, 1996a, p. 313-314).

De ahí se afirma que, desde las conceptualizaciones de Juan Amos Comenio, formuladas en sus obras Didáctica Magna y Pampedia, en el siglo XVII, se ha venido configurando un discurso sobre la pedagogía,

7 Lo escrito entre paréntesis es nuestro. la didáctica y la educación bajo el objeto discursivo y de saber: la enseñanza. Dicho de otra manera, el discurso de Comenio ha logrado individualizar y hacer visible, mediante prácticas y conceptos, un conjunto de conocimientos acerca de la enseñanza. Sin embargo, estos inicios de la pedagogía bajo el objeto de saber, la enseñanza, no comienzan y terminan en Comenio, sino que se extienden hasta Herbart en el siglo XIX. En este periodo comprendido entre Comenio y Herbart, se produjeron opciones de enseñanza, de formación del hombre, de orientación de la escuela, se gestaron modificaciones, y emergieron conceptos y métodos ${ }^{8}$.

De esta manera, la práctica pedagógica ha permitido registrar históricamente la discursividad de la pedagogía, especificando, desde los terrenos arqueológicos, un campo de validez, un horizonte conceptual, un dominio discursivo ${ }^{9}$ para redefinir, reordenar y especificar objetos de saber, nociones, conceptos, métodos y modelos que dan cuenta de la búsqueda de sistematicidad, individualidad y autonomía discursiva de la pedagogía.

Es decir, lo que se pretende al recuperar la práctica pedagógica es, por un lado, aportar consideraciones para un trabajo epistemológico ${ }^{10}$ de la pedagogía a partir de un análisis histórico de esta, y, por el otro, establecer las condiciones en que se le apropia como saber. Por eso, este trabajo de recuperación retoma, en primer lugar, el concepto de "recurrencia" de la historia epistemológica de Canguilhem, no para imponer una normatividad, ni tampoco para hacer el recuento de autores, sino para analizar los actuales problemas y críticas que se le hace a la pedagogía desde diferentes disciplinas o teorías y "para historiar los conceptos, modelos, nociones, obstáculos, reordenamientos discursivos presentes en los procesos

8 Este periodo entre Comenio y Herbart ha sido trabajado a modo de hipótesis por la profesora Zuluaga en los siguientes documentos: "El surgimiento de la pedagogía: Juan Amos Comenio" (1984b); "De Comenio a Herbart" (1989); "Otra vez Comenio" (1992); "Pasado presente de la pedagogía y la didáctica" (1993).

9 Para Foucault, una vez suspendidas las historias continuistas —en nuestro caso la historia de las ideas pedagógicas- se libera todo un dominio constituido por el conjunto de todos los enunciados efectivos, en su dispersión de acontecimientos y en la instancia que le es propia a cada uno: "Antes de habérselas [...] con una ciencia [...] con la obra de un autor [...] con un libro, el material que habrá que tratar en su neutralidad primera es una multiplicidad de acontecimientos en el espacio del discurso en general" (Foucault, 1996a, p. 43).

10 "Epistemología, en este trabajo, se refiere a la historia de las ciencias a partir de las prácticas discursivas, historia que cuenta con herramientas arqueológicas y genealógicas." (Zuluaga, 1999, p. 98). 
de formación de la pedagogía como saber y como disciplina" (Zuluaga, 1987, p. 35). En segundo lugar, se apoya en la historia no del pensamiento, ni mucho menos de las ideas pedagógicas, sino de los discursos para reconocer en la práctica social espacios de formación o de bloqueo epistemológico para una disciplina y, finalmente, se apoya en la pedagogía como saber para analizarla en su ejercicio cotidiano.

Trazar el horizonte conceptual de la pedagogía desde Comenio hasta Herbart fue posible por el trabajo estratégico de la práctica pedagógica, permitiendo delimitar unos campos de saberes y de prácticas articulados por el objeto "enseñanza". Dicho de otra manera, el rescate de la práctica pedagógica permitió ¡volver a los pedagogos!, a la tradición pedagógica para reivindicar la enseñanza como un objeto en el amplio campo de saber de la pedagogía.

En suma, con la práctica pedagógica se logra caracterizar las positividades de la pedagogía en el conjunto de producciones de carácter histórico, conceptual y experimental existente en diversas culturas. Constituye un nuevo escenario histórico y arqueológico para el análisis no solamente de la enseñanza, sino también del maestro, la escuela, el método, el aprendizaje y la formación. También se logra hacer visible una discursividad, que registra no solo objetos de saber sino también nociones, conceptos y modelos que dan cuenta de la búsqueda de sistematicidad de la pedagogía. Además, recuperar la historicidad de la pedagogía a través de la práctica pedagógica permitió realizar un desplazamiento de gran importancia: de la historia de la pedagogía como historia de los autores hacia una historia de los conceptos, prácticas y acontecimientos en torno a la enseñanza. La historia de la pedagogía ya no sería un análisis de los aportes de las figuras "epónimas" de la pedagogía y la educación, sino una historia que registrara las condiciones de posibilidad de la pedagogía como saber y disciplina.

Finalmente, en esta obra encontramos una quinta designación de la práctica pedagógica, que se une a las cuatro designaciones enunciadas ya por la profesora Zuluaga en el documento de $1978^{11}$, la cual

11 Según Zuluaga, "Práctica pedagógica es la noción que ahora ha puesto en juego el Proyecto Filosofía y Pedagogía, es la noción que orienta sus investigaciones acerca de la Pedagogía en Colombia durante el siglo xx. Práctica Pedagógica es una noción que designa: 1. Los modelos pedagógicos, tanto teóricos como prácticos utilizados en los diferentes niveles de enseñanza. 2. Una pluralidad de conceptos pertenecientes a campos heterogéneos de conocimiento, retomados y aplicados por la Pedagogía. 3. Las formas de funcionamiento de los discursos en las instituciones educativas donde se realizan prácticas pedagógicas. 4 . Las características sociales adquiridas por la práctica pedagógica en las instituciones educativas de una sociedad dada que asigna unas funciones a los sujetos de esa práctica" (1978, pp. 9-10). tiene que ver con "Las prácticas de enseñanza en diferentes espacios sociales, mediante elementos del saber pedagógico" (Zuluaga, 1987, p. 196).

Con este nuevo desarrollo de la práctica pedagógica se pretende evitar confusiones entre la práctica científica y la práctica de la enseñanza, entre pedagogía y todas las prácticas de enseñanza, en otras palabras, confundir docencia con pedagogía. Esta nueva alusión indica brevemente

[...] unas diferencias entre esas dos prácticas, diferencias generadas por los procesos de institucionalización de los saberes, pero sobre todo para indicar la diferenciación de por lo menos tres discursos (el de la pedagogía, el de la práctica de la enseñanza y el de la práctica científica), como el lugar de la discusión que debe dar luces a replanteamientos no solo para la práctica de la enseñanza sino también para la pedagogía. (Zuluaga, 1987, p. 67).

\section{Práctica pedagógica: historia de un sujeto de saber}

Sin embargo, el contexto de rescate y recuperación de la práctica pedagógica no se queda aquí, permite acercarnos a la historia de un saber que hace visible un sujeto históricamente definido: "el maestro". Él es que hace posible la enseñanza. Con la recuperación del sujeto del saber pedagógico, a través de la práctica pedagógica, la pedagogía supera esa concepción del pedagogo como "ayo", es decir, como vigilante y acompañante de los niños. Surge una nueva especificación de maestro a partir de la institución y del discurso acerca del método y del niño. Esta nueva figura de maestro es sumamente problemática, pues él ha sido socialmente marginado como portador y productor de saber.

Según Comenio, la misión de este naciente oficio en la escuela "era formar a la juventud" y tener en cuenta "todos los medios de abrir el entendimiento". Este se ejercía desde diferentes prácticas de enseñanza, es decir con diversos métodos, y cada preceptor utilizaba uno distinto para la enseñanza de los saberes. Con la especificación del maestro de escuela surge la necesidad de unificar el método para hacer posible el arte de enseñar todo a todos. Esta unificación del método definió el estatuto social e intelectual del maestro y especificó su oficio hasta entonces amorfo.

Es decir, el método jugó un doble papel: por un lado, se convirtió en un acontecimiento del saber, pues el método como el arte de enseñar todo a todos individualizó el saber pedagógico del maestro; y, por el otro, fue un acontecimiento en el orden del poder, 
porque garantizó el empleo de pocos preceptores, la uniformidad de los contenidos y el control del espacio y del tiempo de la escuela, y la formación y la defensa de los nacientes estados-Nación (Zuluaga, 1984, p. 27).

Ahora bien, esta positividad material e histórica del oficio de maestro también se hizo visible en nuestro país a través de los trabajos de investigación de Martínez, Zuluaga, Echeverri, Quiceno, Sáenz, Saldarriaga y Ospina. Sus estudios se orientan a definir y hacer visibles las configuraciones, las continuidades, las transformaciones y los avatares del oficio de maestro en Colombia desde la Colonia hasta la primera mitad del siglo xx. Su retorno a la institución educativa y al método a través de la práctica pedagógica les permitió recuperar y reconocer al maestro en su experiencia, vivencias y relaciones con la sociedad, el Estado, la ciencia y la cultura.

Dicho de otra manera, las reflexiones sobre el maestro se dieron a partir del viaje del aula a la historia y de la historia al aula. Este tránsito de la escuela a la historia, del encierro de la institución escolar a la exterioridad de lo social, permitió hacer visible al maestro como el sujeto que hace posible la enseñanza, sin restringir su palabra solamente al método, es decir, al conocimiento que habrá de aprender para enseñar, sino lo abre a partir de su oficio de enseñar a las múltiples determinaciones de la sociedad y los saberes. En fin, esta es una historia para la autonomía del maestro porque le propicia identidad en su saber y pluralidad en sus relaciones con los otros saberes y la comunidad intelectual.

\section{Práctica pedagógica: un nudo en una red}

La práctica pedagógica no es una noción metodológica que se explique por sí misma, sino que está en relación con otras nociones metodológicas creadas durante el trabajo de historiar la práctica pedagógica en nuestro país. En primer lugar, aparece la noción metodológica ámbito institucional planteada por el investigador Alberto Martínez Boom. Con esta, se hacen visibles las condiciones que determinaron los inicios de la práctica pedagógica durante la Colonia.

Para Martínez, el ámbito institucional llamado "escuela" permite caracterizar la enseñanza de las primeras letras como una práctica pedagógica con un sujeto específico y un discurso particular. Además, permite señalar las diferencias existentes entre cualquier práctica de enseñanza y la enseñanza de las primeras letras. Según Martínez,
En el Nuevo Reino de Granada [...] el resultado más visible [...] era sin duda la aparición no sólo de un ámbito al que se le reconoce el estatuto de escuela, sino la escuela de primeras letras como realidad tangible, como un tiempo y un espacio donde se congregan diversos sujetos; unos con el carácter de "junta de niños", que asisten con el propósito de "formarse" y otros con el estatuto de maestros, pero no "con un sólo discípulo, haciendo oficio de ayo" ni en condición de "religioso de Orden", sino como hombre libre [...]. (1986, p. 26).

Surge entonces una institución para enseñar definida no por una práctica religiosa, ni por una práctica familiar, sino por una práctica de enseñanza, es decir, aparece un ámbito institucional definido como espacio público, con su horario, un espacio específico (el salón) con sus actividades, un sujeto público (el maestro) y unos destinatarios (los niños). Todos estos elementos definieron la escuela como un ámbito institucional que permitiera hacer visible tanto las relaciones internas del espacio escolar, como las prácticas pedagógicas que establecieron el comienzo histórico del maestro y la escuela en nuestro país.

Instrucción pública es la segunda noción que hace parte de la red nocional de la práctica pedagógica, junto con la noción metodológica planteada por Martínez. La noción de instrucción pública juega un papel estratégico para encontrar las articulaciones entre la práctica política y la práctica pedagógica a nivel de las instituciones, los sujetos y los discursos. Según Echeverri,

La herramienta conceptual más importante, diseñada durante este trabajo, fue la noción de Instrucción Pública. Su valor reside en el análisis, que nos posibilitó, de la Instrucción Pública como acontecimiento bajo la modalidad de una práctica entre prácticas y, en su definición tanto como el territorio donde la práctica pedagógica se inscribe en la práctica política, como un grupo de estrategias mediante las cuales el poder buscó construir un sistema de enseñanza pública que debía delimitar los fines de la Instrucción Pública a los fines de la sociedad. (1989, p. 10).

A través de esta noción, la práctica pedagógica - $\mathrm{y}$ su triada metodológica: institución-sujeto-discursoestablece su vínculo con la práctica política, para hacer visibles las distintas decisiones y tensiones sobre la formación política y moral del ciudadano, sobre la formación de maestros, las escuelas normales y la vigilancia e inspección de la enseñanza en el siglo XIX.

En suma, con la noción instrucción pública la práctica pedagógica es descrita en un espacio fuera de la escuela, en donde miden fuerzas el poder político 
(el Estado) y el poder moral (la Iglesia) para definir su orientación. En este campo de fuerzas se enfrentaron la filosofía escolástica y el benthamismo, las distintas concepciones sobre la escuela y la parroquia, el cura de aldea y el maestro, el Estado y la Iglesia, en fin, diversos forcejeos por la hegemonía de la práctica pedagógica (Zuluaga, 1987).

De otra parte, nos encontramos con la noción apropiación, herramienta metodológica utilizada en el trabajo Mirar la infancia de los investigadores Javier Sáenz, Oscar Saldarriaga y Armando Ospina (1997). En este trabajo,

[...] apropiar un saber es hacerlo entrar en las coordenadas de la práctica social. Es por tanto, un proceso que pertenece al orden del saber como espacio donde el conocimiento está accionado por mecanismos de poder y no por la lógica del movimiento de los conceptos en el conocimiento científico. (p. XIV).

De ahí que

La historia de las prácticas tiene mucho que ver con las particularidades, es decir, la forma como las culturas se apropian de los saberes, la forma como las sociedades organizan sus instituciones y la forma como cada hombre accede a una conciencia colectiva. (Zuluaga, 1997, p. 24).

En conclusión, historiar la práctica pedagógica en nuestro país exigió la construcción de nuevas nociones para estudiar la interioridad de la escuela (he ahí la importancia de la noción ámbito institucional), para analizar la práctica política (por eso la noción instrucción pública), y para estudiar los procesos de apropiación de los conocimientos. Por ello, hemos de comprender la práctica pedagógica como una noción metodológica, la cual no se queda describiendo lo simple o las relaciones internas de la escuela, sino que "abarca lo específico del funcionamiento de la pedagogía y la educación a través de una compleja trama de relaciones con la sociedad" (Zuluaga, 1997, p. XXv) En consecuencia, y siguiendo las reflexiones del profesor Alberto Martínez Boom, uno de los miembros fundadores del GHPP, la práctica pedagógica no es un concepto utilizado por los miembros del grupo de manera cerrada y coherente, sino que, por el contrario, es un concepto alterable, que no es desde hace 39 años sino que deviene (2012). Precisamente esta condición alterada del concepto ha sido una de las condiciones para explorar otras formas de hacer historia, más allá de las ideas pedagógicas, de la educación, de las teorías y políticas educativas, entre otras. En su lugar, se han desarrollado estudios que apuestan por análisis históricos conceptuales (Sáenz, 2003), historia epistemológica de los saberes
(Saldarriaga, 2005), historia de las ciencias de la educación (Rios, 2008), historia de los saberes escolares (Álvarez, 2010, 2013) e historia de la gubernamentalidad o del gobierno de los otros (Noguera, 2012). Describiremos a continuación algunas de ellas:

Nova et vetera (2005), del profesor Oscar Saldarriaga, apuesta por una investigación que indaga por la apropiación de la filosofía neotomista en Colombia. Para Saldarriaga, este estudio desde la historia epistemológica de los saberes permite

\begin{abstract}
Arriesgarnos a estudiar el neotomismo de "modo horizontal". Esto es, comenzar por describir las interacciones que éste estableció con las filosofías contemporáneas que circularon en el campo intelectual colombiano del siglo XIX, en este caso, el sensismo de Destutt de Tracy, el criticismo kantiano, la filosofía escocesabalmesiana del sentido común, el eclecticismo espiritualista de Victor Cousin, el tradicionalismo de De Bonald y de Maistre, el positivismo de Comte y el evolucionismo de Spencer [...]. La historia epistemológica de los saberes propone sacar a la luz los sustratos epistémicos comunes a diversos "sistemas de pensamiento" en un momento dado, sustratos que serían las condiciones de posibilidad, el "a priori histórico", la "matriz de saber", la "ley de coexistencia de un conjunto de enunciados, los principios según los cuales subsisten, se transforman y desaparecen [...]: acontecen" en una formación social y en un momento histórico determinados. (2005, p. XIV).
\end{abstract}

Bien sabemos que la práctica pedagógica supone la educación y el Estado, pero no es reducible a lo que exponga el Estado. En esta ruta, la tesis de Saldarriaga amplia la mirada sobre las relaciones Estado-Iglesia hacia el ámbito de las relaciones escuela-Iglesia-Estado-cultura:

Con ello, se desplaza el centro de interés exclusivo de la descripción en el funcionamiento de la institución eclesial, saltando la frontera disciplinar de la "historia de la iglesia" para cruzarla con otras, en especial con la "historia de la educación y la pedagogía”. En este nuevo marco se sacan a la luz, por un lado, el debate académico que lleva a tomar decisiones sobre los textos de filosofía convenientes para el bachillerato, y por el otro las luchas políticas alrededor de la educación pública y privada. (Saldarriaga, 2005, p. XVI).

Por su parte, Javier Sáenz, en su tesis doctoral (2003), le apuesta a desarrollar un estudio históricoconceptual sobre algunos de los pedagogos fundamentales de Occidente, para analizar el lugar que le asignaron a aquellas dimensiones de la psiquis, como las emociones, los sentimientos, el afecto, los 
instintos y la imaginación, que tanto el pensamiento clásico como el moderno de la pedagogía, la psicología y la filosofía han concebido como no racionales. En ella analiza las formas en las que las prácticas pedagógicas han contribuido a la producción de un individuo dividido dentro de sí mismo. El análisis histórico de la práctica pedagógica en Colombia desarrollado por Sáenz en el libro Mirar la infancia (1997) le permitió mostrar que, por medio del uso de un conjunto de conceptos y prácticas en la educación pública, tuvo lugar un proceso de larga duración denominado "proceso de individualización"12.

En el saber pedagógico colombiano, durante la primera mitad del siglo pasado, este proceso de individualización fue el efecto de un conjunto de discursos y prácticas pedagógicas: fines católicos y prácticas para la configuración de un individuo virtuoso; discursos médicos y prácticas de normalización y regeneración racial de la población; fines económicos de trabajo eficiente y productivo, y de progreso; discursos políticos sobre el orden social, la armonía y la cooperación. También tuvo como efecto un quiebre dentro del mismo sujeto, al intentar aislar y vigilar lo no racional, sometiéndolo al control de la voluntad, la racionalidad y la norma social (Sáenz, 2003).

Finalmente, otros trabajos del GHPP se dieron a la tarea de señalar cómo las prácticas pedagógicas funcionan en diferentes espacios sociales por fuera de la escuela. Entre esos trabajos tenemos, en primer lugar, el escrito por Martínez, Noguera y Castro (1999) sobre la escuela en la Santa Fe colonial. Con esta obra podemos ver cómo desde finales del siglo XVIII la pobreza en Bogotá comienza a ser vista como producto del desorden social y como un peligro potencial para la salud pública. En este sentido, el Estado desarrolla una serie de prácticas "para asegurar el alejamiento, la erradicación o el constreñimiento del desorden impuesto por la miseria" (1999, p. 29), prácticas que desembocaron en varias acciones no solo de vigilancia y control, sino también de formación para la civilidad.

En segundo lugar, en la investigación de Javier Sáenz (2007) titulada Estudio comparativo de las estrategias pedagógicas adelantadas por los tres últimos gobiernos de Bogotá, se examinan las prácticas formativas realizadas por fuera de la escuela durante los gobiernos de Antanas Mockus, Paul Bromberg y

12 Según la definición de Foucault, la individualización es concebida como un proceso que tiene lugar "en la exterioridad del sujeto, el cual describe las formas como la sociedad reconoce y 'recorta' sobre la masa de la población las unidades que la componen". Es la forma en que la sociedad delimita al individuo para actuar sobre él o ella: "El individuo es dividido dentro de sí mismo y separado de los demás. Este proceso lo convierte en un objeto" (1996b, p. 21).
Enrique Peñalosa entre 1995 y el 2003. Con este trabajo el GHPP demuestra cómo las prácticas formativas desarrolladas por estos gobiernos funcionan en una línea de continuidad con las concepciones de la pedagogía escolar y no bajo su opuesto, pero tampoco se muestran como idénticas. En sus propias expresiones,

En términos de su mirada, los tres gobiernos se pueden inscribir en la tradición histórica reseñada en el primer capítulo, de desconfianza hacia la población. Sus enunciados explícitos sobre los aprendizajes deseables en la población son pesimistas; eso es, minimizan las potencialidades de la cultura de la ciudad y se concentran en las actitudes, comportamientos y conocimientos de la población que deben ser creados o corregidos. Y en este sentido, configuran una continuidad con las concepciones de la pedagogía escolar que desde el siglo XVI han señalado la existencia de un mundo corrupto, vicioso, bárbaro e ignorante que debe ser civilizado y transformado de manera radical, formando sujetos para otro mundo, sea este religioso o laico. (Sáenz, 2007, p. 122).

\section{El lugar de la práctica pedagógica en el saber pedagógico}

Según lo anterior, el grupo se reúne en torno a preocupaciones comunes acerca de la historicidad de la pedagogía, la historia de la educación, el análisis de los pedagogos, y la inscripción de sus aportes para tematizar las problemáticas que afectan al maestro, la enseñanza y la escuela, es decir, a los tres elementos constitutivos del maestro, reunidos bajo la práctica pedagógica, que funciona con tres instancias: el sujeto, el saber y la institución, los cuales, a su vez, se inscriben en la noción de saber pedagógico. Sin embargo, la práctica pedagógica juega un papel más de aliada que de subordinada al interior del saber pedagógico.

La caracterización de los procesos de institucionalización del saber pedagógico en nuestra sociedad solo fue posible por el trabajo positivo, estratégico y metodológico de la práctica pedagógica, de ahí que se afirme:

Cuando se demarca el saber pedagógico en una sociedad específica, a partir de la práctica pedagógica, el saber y la práctica se comprometen en los procesos de institucionalización. La institución, el sujeto y el discurso son las instancias metodológicas que pueden aprehender, en la práctica pedagógica, los procesos de institucionalización del saber pedagógico. (Zuluaga, 1987, pp. 54-55). 
Así, la práctica pedagógica se inscribe en el saber pedagógico como una práctica de saber (que involucra las instituciones, los sujetos y los discursos de la pedagogía), permitiendo asumir esta práctica como el conjunto de conocimientos cotidianos o elaborados, conceptos, métodos, prescripciones y observaciones provenientes de la interioridad misma del saber, así como de decisiones externas a él, todos ellos asequibles a la descripción histórica. En consecuencia, la indagación histórica acerca de las prácticas pedagógicas en Colombia ha sido un trabajo metodológico y conceptual que ha posibilitado el develamiento del maestro como sujeto de saber pedagógico, y la postulación de la enseñanza como objeto de saber y práctica de conocimiento, con lo cual se supera su concepción instrumentalista.

Ahora bien, la opción por el saber, para definir el saber pedagógico, está relacionada con razones que hacen de este un espacio amplio y abierto de conocimiento, compuesto por regiones de conceptos con diferente grado de sistematicidad. En este espacio también circulan otros discursos: reglamentos, prácticas, nociones, registros, normas, leyes. Todos estos registros permiten dar cuenta, en el saber pedagógico, de situaciones prácticas y situaciones conceptuales en relación con la enseñanza, la escuela y el maestro. Dicho de otra manera, saber pedagógico evoca pluralidad teórica y diversidad de prácticas, y constitución de campos conceptuales que pueden resolver problemas o avanzar en la comprensión de cuestiones que requieran de combinatorias de conceptos para hacer decible una problemática referida a la escuela, al maestro y a la enseñanza. En resumen, el término saber pedagógico designa de manera general el campo de investigación del Grupo Historia de las Prácticas Pedagógicas en Colombia y también designa el discurso históricamente producido a propósito de la enseñanza. Además, tiene una tarea estratégica y es la de agrupar (no amontonar) los discursos acerca de la enseñanza, trátese de la didáctica, la educación o la pedagogía.

En este sentido, la noción de saber pedagógico, junto con la práctica pedagógica, es la que permite el establecimiento de un mayor número de relaciones entre la pedagogía (su historicidad y su surgimiento como disciplina) y otras prácticas en las cuales circulan conceptos y objetos pedagógicos. Afirmamos junto con la práctica pedagógica porque ella tiene que ver con el saber, la formación y la enseñanza. Esta última no se da solamente en el aula sino en otros espacios sociales. De esta manera, se puede seguir afirmando, como hipótesis de trabajo, que hoy en día el lugar de elaboración de la pedagogía ya no sea solamente la escuela sino la ciencia (Zuluaga, 1993).
Lo anterior no significa coincidir con las teorías desescolarizantes en la necesidad de clausurar las escuelas, sino que la institución escolar ya no es el único espacio que integra las conceptualizaciones entorno a la enseñanza. Por ejemplo, para que la didáctica de cada ciencia tome su senda y se desarrolle en la actualidad, no es condición de posibilidad la existencia de la escuela. Es decir, la recuperación de la práctica pedagógica que permite entender la pedagogía como saber no crea un dominio o espacio discursivo hegemónico, cerrado sobre sí mismo, sino, por el contrario, genera un espacio autónomo y creador para la pedagogía en un dominio singular (la enseñanza). Además, este espacio conquistado le permite a la pedagogía relacionarse con otros dominios discursivos.

¿Por qué la enseñanza? Porque ha sido ella, durante la historia del saber pedagógico, la que ha tenido más relaciones con otros conceptos, ha generado o hecho parte de una práctica (la práctica de la enseñanza o práctica pedagógica), y la que ha sufrido modificaciones, según las teorías que hayan desarrollado, cuestionado o delimitado el saber pedagógico. Es un objeto de saber que ha permanecido individualizando siempre una práctica en la que siguen jugando el maestro, la escuela, el conocimiento y el hombre. Si hacemos la historia del concepto de enseñanza, en la perspectiva de los saberes y bajo la noción práctica pedagógica, ella nos permitirá hacer visibles las diversas acciones que se derivan de la teoría y los cambios prácticos que posibilitan las nuevas teorías o modificaciones conceptuales.

En este sentido, la práctica pedagógica sustentada en el saber pedagógico posibilita relaciones múltiples de la pedagogía con otros saberes, y nos indica de qué manera las nuevas miradas y modificaciones conceptuales sobre el maestro, el alumno y el maestro surgidas en las diferentes disciplinas que han contribuido tradicionalmente al enseñar (psicología, sociología, entre otras) delimitan, adecuan y reordenan el espacio de saber llamado saber pedagógico. Es decir, con la práctica pedagógica entendida como práctica de saber podemos tanto visibilizar nuevas regiones de conceptos y problemáticas referidas a la enseñanza, como señalar cuáles han sido las continuidades y transformaciones conceptuales y prácticas que ha producido la teoría cognitiva, las ciencias de la educación, las investigaciones sobre enseñanza de las ciencias y la teoría curricular al interior del saber pedagógico. Por ejemplo: ¿qué reordenamientos produce, a nivel del saber y la práctica pedagógica, la llamada "teoría de las inteligencias múltiples" de Howard Gardner, la transposición didáctica de Chevallard, y las didácticas disciplinares?, ¿qué nuevas 
relaciones y transformaciones se producen con el concepto de aprendizaje? Estos son interrogantes que todavía siguen aguardando una reflexión en el espacio del saber pedagógico.

\section{Práctica pedagógica: nuevas lecturas y usos}

En este apartado quisiera ubicar la reflexión sobre los nuevos horizontes y perspectivas de análisis de la práctica pedagógica. Es innegable para muchos de los investigadores del GHPP la pertinencia de la noción práctica pedagógica al igual que la del saber pedagógico, elaboradas por la profesora Zuluaga para pensar la pedagogía en Colombia. Sin embargo, se consideran algunos desplazamientos (¿con distancia?) respecto a la noción práctica pedagógica, según como lo afirman las siguientes expresiones:

\begin{abstract}
Aunque las nociones de saber pedagógico y práctica pedagógica han sido fundamentales en los trabajos del GHPP, deben considerarse ciertos desplazamientos y actualizaciones que algunos trabajos han operado en ellas. Por un lado, la participación de integrantes del grupo en la reestructuración de escuelas normales en Antioquia desde finales de la década de 1990, posibilitó la construcción de la noción campo conceptual y narrativo de la pedagogía (CCNP) (Echeverri, 2015), con la que se tomó distancia de la idea de pedagogía como disciplina en formación. Por el otro, a partir de la lectura de los cursos ofrecidos por Michel Foucault en el Collège de France se ha explorado una entrada a la práctica pedagógica que, sin abandonar la tríada saber/sujeto/institución, la comprende como práctica de gobierno, vinculada al problema de la conducción de la conducta individual y colectiva (Noguera, 2012; Marín, 2015b; León, 2012). (Parra, 2017, p. 98).
\end{abstract}

Como ya lo había expresado Zuluaga, la noción práctica pedagógica no se agota en sí misma, sino que está en relación con otras nociones metodológicas creadas durante el trabajo de historiar la práctica pedagógica en nuestro país.

Además, con la noción práctica pedagógica ha sido posible analizar los métodos y las técnicas de enseñanza desde el siglo XIX en Colombia, no como un método al margen de la dominación y el gobierno de los otros, sino "hasta en sus formas más meticulosas de organización escolar la forma como las clases dominantes sometían el cuerpo, el conocimiento y la moral de los niños a un aprendizaje para la obediencia y el sometimiento" (Zuluaga, 1978, p. 13).
Al igual que las prácticas de gobierno, la práctica pedagógica se refiere a múltiples espacios de saber e incumben a muchos sujetos: padres de familia, inspectores, curas, pedagogos y al maestro en relación con sus alumnos. Dicho esto, vemos que la práctica pedagógica tiene la potencia de hacer visibles tanto procesos de subjetivación, como procesos de invidualización ${ }^{13}$. Por tanto, la práctica pedagógica concibe diversas subjetividades respecto a las cuales el sujeto maestro en relación con sus alumnos no es más que una modalidad de práctica de gobierno. Ciertamente, la tarea de recuperar la historia de la práctica pedagógica en Colombia significó recuperar a un sujeto ignorado por los historiadores de la educación, la cultura y la sociedad en general: el maestro, pero no como un sujeto singular que trasciende a los otros sujetos que están relacionados y comprometidos con la escuela, la enseñanza y la pedagogía. En las expresiones de Zuluaga, con la noción práctica pedagógica se imprimió a la investigación sobre la pedagogía en Colombia, de un lado, su objeto de análisis:

Lo pedagógico como teoría y como práctica. Lo pedagógico como lo que permite a los maestros entrar en relación con el conocimiento. Lo pedagógico como acontecimiento social susceptible de ser escrito en su especificidad (no trascendente) histórica. Estas investigaciones acerca de la práctica pedagógica, se inscriben en un horizonte general que bien puede llamarse: las condiciones históricas culturales del maestro, condiciones que solo podrán conocerse a través de la historia de nuestra práctica pedagógica. Pero es también un punto de ese horizonte general, la pregunta por el papel social asignado al maestro como "agente de la cultura". Al maestro se le ha declarado "transmisor de conocimientos", pero a la vez se le ha negado su acceso a la cultura. ¿Quién es el maestro? ¿Quién, entre todos los sujetos de una sociedad dada, es un maestro?". (Zuluaga, 1978, p. 10).

De otro lado, y en coherencia con la pregunta sobre quién es el maestro, se logró hacer una distinción entre los sujetos de la enseñanza, a saber:

Por una parte aquel que se relaciona con las ciencias o con los saberes a partir de un método, es decir,

13 Siguiendo los trabajos de Sáenz et al. (1997), el análisis histórico de la práctica pedagógica en Colombia durante la primera mitad del siglo xx mostró que por medio del uso de un conjunto de conceptos y prácticas en la educación pública tuvo lugar un proceso de larga duración de individualización. Este fue el resultado de un conjunto de discursos y prácticas pedagógicas: fines católicos y prácticas para la formación de un individuo virtuoso; discursos médicos y prácticas de normalización y regeneración racial de la población; fines económicos de trabajo eficiente y productivo, y de progreso; discursos políticos sobre el orden social, la armonía y la cooperación. 
el maestro. Porque el ejercicio de su saber está completamente fetichizado desde una concepción instrumental del método de enseñanza. [...] Pero existe por otra parte otro sujeto que también enseña y al que se llama docente. Este sujeto de la enseñanza es reconocido como tal, no a partir del método de enseñanza, sino del saber que transmite; el puede ser profesor de matemáticas, profesor de física, profesor de filosofía, profesor de sociología, es decir, su estatuto como docente, en la sociedad se le reconoce desde otro saber que no es la Pedagogía. Sin embargo, los dos sujetos enseñan, pero la diferencia entre esos dos sujetos es una resultante de la forma de institucionalización y de la adecuación social de los saberes, más no es una distinción que se derive de la naturaleza de la Pedagogía. (Zuluaga, Echeverri y Quiceno, 1999, p. 49).

Entre el 2012 y el 2014 el proyecto "Saberes y disciplinas escolares en Colombia" parte de una problematización de la imagen de la pedagogía reducida al método de enseñanza, de modo que saberes y disciplinas escolares se examinaran como dimensiones de la práctica pedagógica y del saber pedagógico que, mediante la relación pedagogía/ciencias/saberes, nos conduce a pensar los saberes y las disciplinas como formas de apropiación de la ciencia y los saberes en la escuela. Aquí la práctica pedagógica no solamente haría visibles procesos de institucionalización y funcionamiento de la ciencia y los saberes en las instituciones educativas, sino también comprendería las formas de enunciación, circulación y apropiación de los saberes enseñados (Rios, 2015). Con esta investigación la práctica pedagógica activa su propósito originario, a saber, epistemológico, estratégico y político, lo cual nos llevaría a tomar dos vías de proyección: la primera proponer

[...] que la práctica pedagógica es mucho más que «lo que el maestro hace en el aula», se señala algo que pareciera evidente, pero que no lo es en absoluto: que el maestro actúa no solo en su salón, sino en la escuela, y la escuela actúa a su vez en las comunidades y en sus territorios. (Saldarriaga, 2016, p. 14).

En medio de los actuales procesos de desescolarización de la pedagogía, la segunda vía sería:

Es oportuno preguntarnos por lo que le queda a la escuela de pedagogía. Siempre hemos dado por hecho que la práctica pedagógica, y en consecuencia el saber pedagógico, han trascendido a la escuela, como explicábamos antes, pero también es cierto que siempre hemos querido preguntarnos por el lugar de la escuela y del maestro en medio de dicha práctica. Ese es quizás la clave de los aportes del GHPP, que pone en relación la escuela y el maestro con la práctica pedagógica y el saber pedagógico, asumidos como un acontecimiento cultural, social, político, ético y estético más amplio. Pero entonces, ¿qué le queda hoy de pedagogía a la escuela y al maestro, en medio de la sociedad del conocimiento, del educapital, de la sociedad del aprendizaje, de la autoayuda, de los procesos de subjetivación juveniles y de las pedagogías indígenas, comunitarias, populares, feministas y demás? (Álvarez, 2015, p. 24).

En suma, podríamos afirmar, junto con Saldarriaga (2016), que la noción práctica pedagógica, por un lado, se ha enrarecido, y, por el otro, al decir de Martínez, ha devenido, es decir, ha seguido nuevos modos de problematización de las escisiones entre teoría y práctica, y todos los juegos de oposición que las siguen configurando.

\section{A modo de cierre}

Para finalizar, planteamos algunas reflexiones que podrían abrir la posibilidad de un nuevo programa de investigaciones:

1. La práctica pedagógica no es una práctica que hegemoniza toda práctica, es decir, religiosa, familiar, social, política o comunitaria, con solo pensar que cada una de ellas lleva una intencionalidad formativa. Es una práctica que analiza la interioridad y la exterioridad de la escuela. Por eso, la práctica pedagógica descrita por el grupo de investigación no fue ajena a las prácticas políticas y sociales de la época estudiada. En otros términos, la práctica pedagógica es una práctica que se analiza en un conjunto de relaciones complejas. Cualquier práctica por sí misma no es una práctica pedagógica, cuando ella está referida a la enseñanza lo hace dentro de un espacio de saber específico.

2. La práctica pedagógica no separa, ni clasifica las diferentes prácticas, ni tampoco las uniformiza, sino las relaciona dentro de un espacio de saber, un dominio discursivo específico, es decir, en un campo de regulaciones, pues la práctica pedagógica no es anómica, es regulada y articulada por el objeto de enseñanza. Es una práctica atravesada por varias prácticas, de ahí la importancia de seguir afirmando que la práctica pedagógica comprende los modelos pedagógicos tanto teóricos como prácticos utilizados en los diferentes niveles de enseñanza.

3. La práctica pedagógica permite preguntar y diferenciar quién habla, desde dónde habla y desde qué discurso lo hace. Por eso, solo en la práctica pedagógica el sujeto de saber que enseña la biología, la física, la química, entre otros saberes, tiene la posibilidad de que su práctica de saber sea reconocida como una 
práctica pedagógica, como una práctica de enseñanza dentro de un espacio de saber específico. Entonces, "hacer historia es también luchar por ganar una presencia en el espacio social del conocimiento" (Zuluaga, 1997, p. 24). En esta ruta el proyecto de investigación "Saberes y disciplinas escolares en Colombia”, desarrollado por varios miembros del GHPP, apuesta por una historia de la enseñanza de los contenidos disciplinares y de los saberes en la escuela, para hacer visibles los diversos procesos de saber que ocurren en la enseñanza. Junto con las tradiciones abiertas por André Chervel y Dominique Julia en Francia, las conceptualizaciones de Olga Lucia Zuluaga respecto a la pedagogía en Colombia, y la desarrollada por el norteamericano John Dewey, este proyecto problematiza, apoyado en un amplio y diverso archivo documental, que las disciplinas, en el contexto de la enseñanza, no son simples "metodologías", ni mucho menos que la pedagogía funciona como un lubricante encargado de engrasar los mecanismos de la máquina de la enseñanza. En el marco de esta problematización, el proyecto asume la pedagogía como práctica relacionada con el discurso, de ahí que el GHPP no ha hecho, ni hará historia de la enseñanza en abstracto, sino una historia de prácticas de saber localizadas en unas condiciones específicas, singulares, raras.

El proyecto del grupo es hacer historia de las prácticas pedagógicas, no de la educación y la pedagogía en abstracto. Su apuesta ha sido desde hace más de treinta años pensar la pedagogía en Colombia a través del concepto práctica pedagógica, para hacer visible su relación con las ciencias, la ciudad, las comunidades, la cultura y el pensamiento.

\section{Referencias}

Álvarez, A. (2010). Formación de nación y educación. Bogotá: Siglo del Hombre.

Álvarez, A. (2013). Las ciencias sociales en Colombia. Genealogías pedagógicas. Bogotá: IDEP.

Álvarez, A. (2015). Del saber pedagógico al saber escolar. Pedagogía y Saberes, 42, 21-29.

Echeverri, A. (1989). Santander y la instrucción pública, 1819-1840. Bogotá: Foro Nacional por Colombia/ Universidad de Antioquia.

Foucault, M. (1996a). La arqueología del saber. México D.F.: Siglo XXI.

Foucault, M. (1996b). Tecnologías del yo y otros escritos afines. Barcelona: Paidós.
Martínez, A. (1986). Escuela, maestro y métodos en Colombia, 1750-1820. Bogotá: Universidad Pedagógica Nacional.

Martínez, A. (2012). Práctica pedagógica: historia y presente de un concepto. En D. F. Barragán, A. A. Gamboa y J. E. Urbina (Eds.), Práctica pedagógica. Perspectivas teóricas (pp. 55-72). Bogotá: ECOE.

Martínez, A., Noguera, C. y Castro, J. (1999). Maestro, escuela y vida cotidiana en Santafé colonial. Bogotá: Sociedad Colombiana de Pedagogía.

Noguera, C. (2012). El gobierno pedagógico: del arte de educar a las tradiciones pedagógicas modernas. Bogotá: Siglo del Hombre/Grupo de Historia de la Práctica Pedagógica.

Parra, G. (2017). Matemáticas como saber escolar en Colombia (1845-1906): gobierno, razón y utilidad. Pedagogía y Saberes, (47), 95-106.

Ríos, R. (2008). Las ciencias de la educación en Colombia. Bogotá: Magisterio.

Ríos, R. (2015). Historia de la enseñanza en Colombia: entre saberes y disciplinas escolares. Pedagogía y Saberes, $42,9-20$.

Sáenz, J. (2003). Pedagogical discourse and the constitution of the self (tesis inédita de doctorado). University of London, Institute of Education, Londres, Inglaterra.

Sáenz, J. (2007). Desconfianza, civilidad y estética: las prácticas formativas estatales por fuera de la escuela en Bogotá, 1994-2003. Bogotá: Universidad Nacional de Colombia/IDEP.

Sáenz, J., Saldarriaga, O. y Ospina, A. (1997). Mirar la infancia: pedagogía, moral y modernidad en Colombia, 1903-1946. Medellín: Universidad de Antioquia/Foro Nacional por Colombia/Clío.

Saldarriaga, 0. (2005). Nova et vetera o de cómo fue apropiada la filosofía neotomista en Colombia, 1868-1930. Catolicismo, modernidad y educación desde un país poscolonial latinoamericano (tesis inédita de doctorado). Université Catholique de Luovain, Lovaina, Bélgica.

Saldarriaga, O. (2016). La escuela estallada: diálogos entre dos nociones de práctica pedagógica. Memoria y Sociedad, 20(41), 10-20.

Zuluaga, O. (1976). Filosofía y pedagogía. Medellín: CIED.

Zuluaga, O. (1977). Didáctica y conocimiento. Medellín: CIED.

Zuluaga, O. (1978). Colombia: dos modelos de su práctica pedagógica durante el siglo XIX. Medellín: Universidad de Antioquia, CIED. 
Zuluaga, O. (1984a). El maestro y el saber pedagógico en Colombia, 1821-1848. Medellín: Universidad de Antioquia/CIED.

Zuluaga, O. (1984b) El surgimiento de la pedagogía: Juan Amós Comenio. Revista Sociología, (6-7), 45-52.

Zuluaga, O. (1989). De Comenio a Herbart. Educación y Cultura, (17), 45-51.

Zuluaga, 0. (1992). Otra vez Comenio. Educación y Pedagogía, (8-9), 241-273.

Zuluaga, O. (1993). Pasado presente de la pedagogía y la didáctica. En Objeto y método de la pedagogía (pp. 119125). Medellín: Universidad de Antioquia.
Zuluaga, O. (1997). Los conceptos y las prácticas: una estrategia para hacer historia de la pedagogía. En Encuentro de Investigadores Distritales en Educación. Bogotá: IDEP.

Zuluaga, O., Echeverri, A. y Quiceno, H. (1999). Conceptualizaciones del proyecto historia de la práctica pedagógica en Colombia. Documento en pdf.

Zuluaga, O. (1999). Pedagogía e historia. Bogotá: Siglo del Hombre, Anthropos, Universidad de Antioquia. 